\title{
Optimal drug-eluting stent implantation with the aid of optical coherence tomography in the stenotic lesion of ectatic coronary artery
}

\author{
Yongcheol Kim, Myung Ho Jeong, Min Chul Kim, Doo Sun Sim, \\ Young Joon Hong, Ju Han Kim, Youngkeun Ahn \\ Division of Cardiology, Chonnam National University Hospital, Gwangju, Korea
}

A 59-year-old man presented with 1 day history of intermittent angina and an elevated high sensitivity troponin measurement. He had received stent implantation with a $4.5 \times 16 \mathrm{~mm}$ bare metal stent (BMS) at the proximal portion of the ectatic right coronary artery (RCA) 3 years prior to this. Urgent coronary angiography (CAG) demonstrated the severe stenosis in the distal RCA with patent of implanted BMS (Fig. 1A). The selection of appropriate stent size and stent optimization to avoid under-expansion and malapposition due to the stenotic lesion of ectatic RCA led to an assessment with optical coherence tomography (OCT). OCT (Suppl. Video 1) demonstrated that distal reference lumen and external elastic lamina (EEL) diameter of $4.67 \mathrm{~mm}$ and $5.20 \mathrm{~mm}$, respectively (Fig. 1B). OCT also showed a minimal lumen area of $3.32 \mathrm{~mm}^{2}$ (Fig. 1C) and proximal reference lumen and EEL diameter of
$3.91 \mathrm{~mm}$ and $4.65 \mathrm{~mm}$, respectively (Fig. 1D). The OCT assessment led to predilation with a $3.0 \times$ $\times 15 \mathrm{~mm}$ balloon, and a $4.0 \times 24 \mathrm{~mm}$ bioabsorbable polymer everolimus-eluting stent (SYNERGY ${ }^{\mathrm{TM}}$, Boston Scientific, USA) implantation at 16 atmosphere. Postdilation was achieved with a $5.0 \times 12 \mathrm{~mm}$ noncompliant balloon at up to 18 atmosphere in the distal portion of implanted drug eluting stent (DES). Repeated OCT assessment demonstrated (Suppl. Video 2) a minimal stent area of $11.92 \mathrm{~mm}^{2}$ (Fig. 1E), excellent stent expansion, and good strut apposition without edge dissection. Final CAG showed good distal flow without residual stenosis (Fig. 1F).

Percutaneous coronary intervention with DES for ectatic coronary artery is challenging due to stent optimization. This case highlights the important role OCT plays in the stent optimization for ectatic vessel.

Conflict of interest: None declared

Address for correspondence: Myung Ho Jeong, MD, Cardiovascular Convergence Research Center of Chonnam National University Hospital, Gwangju 501-757, Republic of Korea, tel: 82-62-220-6243, fax: 82-62-228-7174, e-mail: myungho@chollian.net

Received: 24.11.2017 Accepted: 25.12.2017 


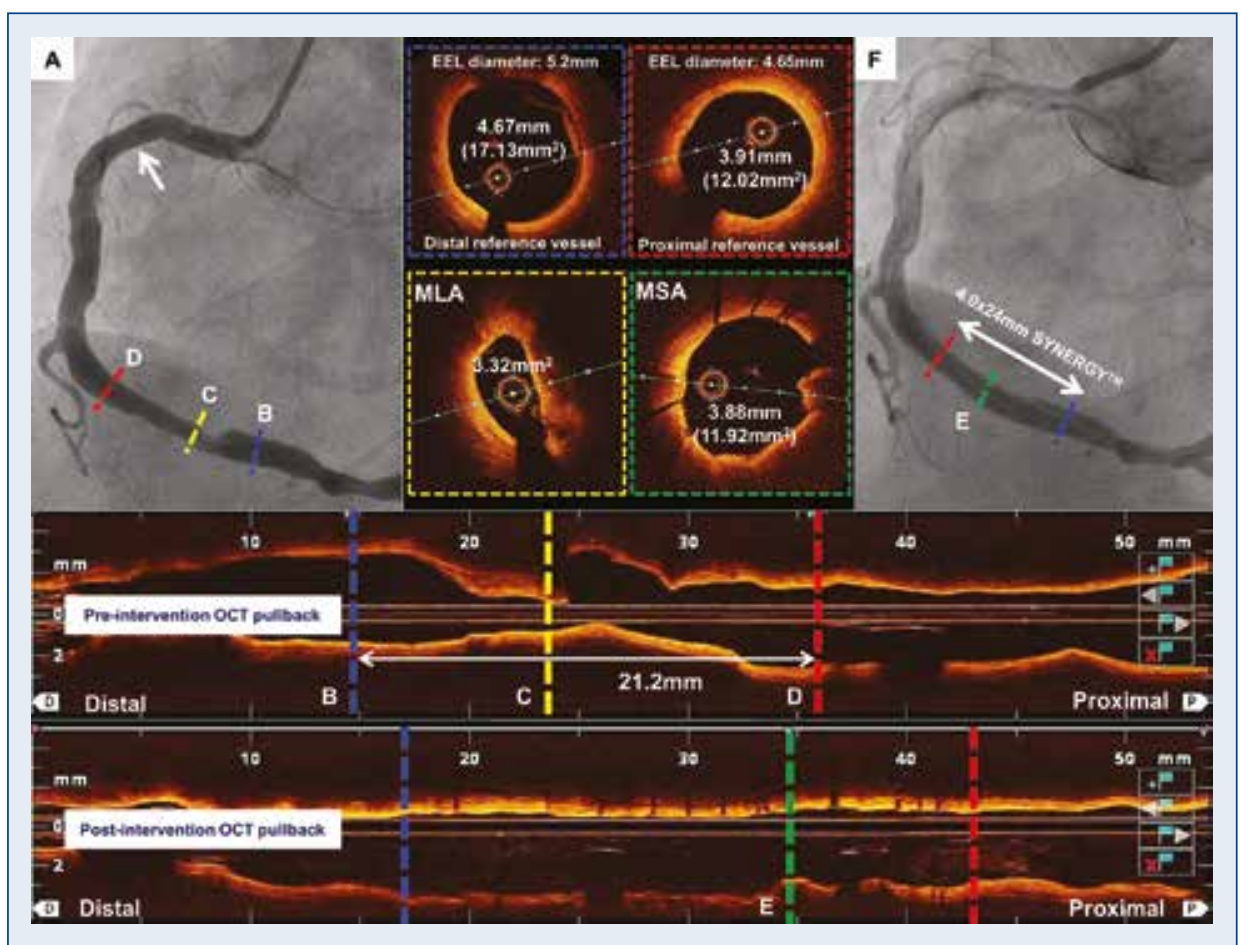

Figure 1. A. Angiographic assessment demonstrating severe stenosis in the distal right coronary artery (RCA) with ectasia and patent bare metal stent (BMS) in the proximal RCA (white arrow); B. Optical coherence tomography (OCT) assessment demonstrating distal reference lumen diameter of $4.67 \mathrm{~mm}$ and external elastic lamina (EEL) diameter of $5.20 \mathrm{~mm}$; C. OCT cross-section demonstrating minimal lumen area (MLA) of $3.32 \mathrm{~mm}^{2}$; D. OCT demonstrating proximal reference lumen and EEL diameter of $3.91 \mathrm{~mm}$ and $4.65 \mathrm{~mm}$, respectively; $\mathbf{E}$. OCT demonstrating minimal stent area (MSA) of $11.92 \mathrm{~mm}^{2}$ and good stent apposition; F. Final angiogram demonstrating good distal flow without residual stenosis from treatment with $4.0 \times 24 \mathrm{~mm}$ SYNERGYTM stent. 